Please do not remove this page

RMIT

UNIVERSITY

\title{
Three-dimensional ionospheric tomography by an improved algebraic reconstruction technique
}

Wen, Debao; Yuan, Yunbin; Ou, Jikun; Huo, Xingliang; Zhang, Kefei

https://researchrepository.rmit.edu.au/esploro/outputs/9921861809101341/filesAndLinks?institution=61RMIT_INST\&index=null

Wen, D., Yuan, Y., Ou, J., Huo, X., \& Zhang, K. (2007). Three-dimensional ionospheric tomography by an improved algebraic reconstruction technique. GPS Solutions, 11(4), 251-258.

https://doi.org/10.1007/s10291-007-0055-y

Document Version: Accepted Manuscript

Published Version: https://doi.org/10.1007/s10291-007-0055-y

Repository homepage: https://researchrepository.rmit.edu.au

(C) Springer-Verlag 2007

Downloaded On 2023/04/26 21:38:18 +1000 
Citation: Wen, D, Yuan, Y, Ou, J, Huo, X and Zhang, K 2007, 'Three-dimensional ionospheric tomography algebraic reconstruction technique', GPS Solutions, vol. 11, pp. 251-258.

\title{
Three-dimensional ionospheric tomography by an improved algebraic
}

\section{reconstruction technique}

\author{
Wen Debao ${ }^{1,2}$ Yuan Yunbin $^{1}(\bowtie)$ Ou Jikun ${ }^{1}$ Huo Xingliang ${ }^{1,2}$ and Zhang Kefei ${ }^{3}$ \\ 1 Institute of Geodesy and Geophysics, Chinese Academy of Sciences, Wuhan 430077, China; \\ 2 Graduate School of Chinese Academy of Sciences, Beijing, 100039, China; \\ 3. School of Mathematical and Geospatial Sciences, RMIT University, Australia \\ Email: yybgps@asch.whigg.ac.cn \\ Telephone: 086-027-68881072 \\ Fax: 086-027-68881362
}

Abstract: In this paper an improved algebraic reconstruction technique (IART) is presented for the tomographic reconstruction of ionospheric electron density (IED). This method applies the total electron content (TEC) data measured by an ionospheric tomography system to invert the spatial distribution of IED from a set of apriori IED distributions. In this method, a data-driven adjustment of the relaxation parameter is performed to improve the computation efficiency and image quality of the classical algebraic reconstruction technique (ART). In addition, the new algorithm is also combined with ionospheric space discretization technique and CHAMP occultation data to improve the vertical resolution. A numerical simulation experiment based on the known positions of GPS satellites and ground receivers is carried out to validate the reliability of the new method. It is then applied to the inversion of IED from real GPS data. Inverted results show that the IART algorithm has better sufficiency (accuracy?) and efficiency than the conventional ART algorithm. The reliability of the IART algorithm is also validated by ionosonde data recorded at Wuhan station.

Keywords: GPS; computerized ionospheric tomography (CIT); total electron content (TEC); algebraic reconstruction technique (ART), ionospheric electron density (IED).

\section{Introduction}

Ionospheric TEC is an important physical parameter of the ionosphere, which can be measured by the received dual-frequency radio signals passing through the ionosphere from satellite systems such as GPS. Since ionospheric TEC is the line integral of IED along the ray path from a satellite to a receiver, TEC data from multi-station can be usually applied to study the horizontal variations of ionospheric structure. However, it is disadvantageous to monitor the vertical variations of ionospheric structure because the conventional approaches to measure TEC cannot derive the IED distribution along a ray path. Therefore, imaging ionospheric electron densities from real satellite radio signals has become an indispensable technique for many scientific and engineering applications.

Austen et al (1986) first proposed the CIT technique and demonstrated its feasibility to reconstruct IED distribution by using TEC data. Various theoretical studies (Raymund et al, 1990; Na and Lee, 1990; Fremouw et al. 1992) and experimental observations (Andreeva et al. 1990; Kunitsyn and Tereshchenko, 1992; Pryse and Kersley, 1992) have been carried out since then. In these studies, however, only radio signals from the Navy Navigation Satellite System (NNSS) received by several ground stations are applied. Although they are helpful for understanding the vertical structure of the ionosphere, only two-dimensional ionospheric image at a fixed longitude chain can be obtained (Ma and Maruyama, 2005). In addition, since 
the temporal and spatial coverage of the NNSS observations is limited, the recovered IED profile can not be obtained with ideal temporal-spatial resolution. The advent of GPS has opened a new avenue for monitoring and investigating ionospheric activities due to its advantages such as high-precision, near real-time availability and high-resolution. The establishment of more and more global and regional ground-based GPS networks (e.g. the international GNSS service (IGS)) and space-based GPS observations (e.g. CHAMP occultation data) makes it possible to reconstruct three-dimensional image of the IED. Up to now, GPS-based tomographic investigations have been extensively studied (e.g. Hajj et al. 1994; Hansen et al. 1997; Leitinger et al. 1997; Rius et al. 1997; Howe et al. 1998; Hernandez-pajares et al. 1998; Bust et al. 2000; Stoll et al.2003; Yin et al. 2004; Ma and Maruyama, 2005; Yizengaw et al. 2005).

For GPS-based CIT, it has been found that the reconstructed images of the IED are usually distorted. The reasons are as follows: first, the number of ground receivers is usually limited, and they don't distribute evenly; second, horizontal ray paths in satellite-to-receiver geometry, which are very important to improve the vertical resolution of ionospheric tomography, are absent. Therefore, the imaging quality of the IED needs to be effectively advanced.

To solve the above problems, numerous algorithms have been presented in recent years. As a whole, they can be divided into two categories: one is the iterative reconstruction algorithm such as the ART; the other is the non-iterative reconstruction algorithm such as the singular value decomposition (SVD) (Wu et al. 2000). For an ionospheric tomography system, which needs high temporal-spatial resolution, the projection matrix that needs to be inverted is usually very large. This makes it difficult to invert the matrix. In this case, SVD technique is not applicable. Although some researchers (e.g. Kunitake et al. 1995; Ruffini et al. 1998) have applied SVD technique to reconstruct three-dimensional tomographic images of the ionosphere, real temporal-spatial resolution of the recovered IED profiles are limited. On the other hand, it has been demonstrated that the ART can provide better reconstruction from either very few or limited views (Andersen, 1989; Oskoui and Stark, 1989), and it is especially suitable for the inversion of IED with high temporal-spatial resolution. However, its disadvantage is the heavy computation power requirement. Therefore, it is necessary to develop a three-dimensional ionospheric tomgoraphy method with high-resolution and high-efficiency. For this purpose, a new ionospheric tomography algorithm is presented and validated in this paper. The algorithm is an improved ART, called the IART, which improves both computation efficiency and imaging quality by introducing an adaptive adjustment to relaxation parameters during the inversion process. In addition, to improve the vertical resolution both ionospheric region discretization technique and space-based CHAMP occultation data are combined with the IART to jointly perform the inversion of IED. Numerical simulation experiments based on the known positions of a set of GPS satellites and ground receivers show that IED images can be reconstructed in three-dimensional mode by using the IART with both high reconstruction quality and computation efficiency. Then it is successfully applied to the IED inversion from real GPS observation data, and the reliability of the inverted results is also validated by ionosonde observation data recorded at Wuhan station.

\section{Tomographic formulation}

As mentioned above, ionospheric slant TEC (STEC) is the line integral of IED along the ray path from a satellite to a receiver, and it can be defined as:

$$
S T E C=\int_{l} N e(s) d s
$$


where $N(s)$ (this is not exactly the same with $\mathrm{Ne}(\mathrm{s})$ ???) represents the IED, and $l$ is the ray propagation path of each GPS satellite-receiver pair.

From equation (1), it can be seen that the relation between ionospheric STEC and IED is not linear. For the simplicity of IED inversion, an imaged region of the ionosphere is first discretized into some small pixels in a selected reference frame. Within each pixel, the electron density can be assumed constant. The STEC along the ray path can then be expressed as a finite sum of shortest integrals in all segments of the ray path. Each set of TEC values along the ray path from a satellite to a receiver can be written as:

$$
\mathrm{STEC}_{\mathrm{i}}=\sum_{j=1}^{n} A_{i j} x_{j}+e_{i}
$$

Equation (2) can be generally written in a simple matrix notation as:

$$
y_{m \times 1}=A_{m \times n} x_{n \times 1}+e_{m \times 1}
$$

where $n$ is the number of pixels in the image, $m$ is the number of STEC measurements, $y$ is a column vector of the $m$ known STEC measurements, $A$ is an $m \times n$ matrix with $A_{i j}$ being the length of ray $i$ traversing pixel $j, x$ is a column vector consisting of all the unknown electron densities in all the pixels, and $e$ is a column vector associated with the discretization errors and measurement noises.

Ionospheric tomography is an inversion technique whereby a series of IED profiles can be recovered from a set of TEC measurements derived from the received GPS signals at ground-based and space-based GPS receivers. Thus the inversion precision of the IED mainly depends on the quality of the available TEC measurements and the performance of the selected reconstruction algorithm. Methods for computing STEC from GPS observations have been described in several papers (Klobuchar, 1991; Sardon et al., 1994; Breed et al., 1996). More specifically, Horvath and Essex (2003) have clearly outlined the method used in this paper. Hence, the focus of this work is the improved reconstruction algorithm only.

\section{An improved algebraic reconstruction technique (IART)}

The ART is a conventional algorithm in solving equation (3), and the procedure can be described as follows:

$$
x^{(k+1)}=x^{(k)}+\lambda_{k}\left(y_{i}-a_{i} x^{(k)}\right)
$$

and the column vector $\lambda_{k}$ consisting of all relaxation parameters is given as:

$$
\lambda_{k}=\gamma \cdot a_{i}^{T} /\left(a_{i} a_{i}^{T}\right)
$$

where $a_{i}$ represents the ith row vector in projection matrix A, $\gamma$ is called relaxation parameter which is fixed in an ionospheric tomography system. From equations (4) and (5), it can be seen that how to determine column vector $\lambda_{k}$ is very important for the ART. Effective determination of the column vector 
$\lambda_{k}$ is advantageous for the reconstruction of IED with high-resolution. According to equation (5), $\lambda_{k}$ only depends on $\gamma$ because vector $a_{i}$ does not vary with the inverted process of IED. It is noted that the column vector $\lambda_{k}$ consisting of a set of small relaxation parameters can usually reduce the artifacts (artificial????) noise in ART, but the disadvantage is the degradation of computation efficiency. It is therefore necessary to improve the conventional ART in order to image IED with high-efficiency and high-quality. This means that the column vector $\lambda_{k}$ should be adaptively adjusted in an iterative process

so that all relaxation parameters in vector $\lambda_{k}$ become gradually smaller with the increase of the number of iterations, and the convergence can be improved. In this paper, the column vector $\lambda_{k}$ in equation (5) will be improved as follows:

$$
\lambda_{k}=g^{(k-1)} /\left(a_{i} \cdot g^{(k-1)}\right)
$$

where $g^{(k-1)}=\left[g_{1}^{(k-1)}, g_{2}^{(k-1)}, \cdots, g_{n}^{(k-1)}\right]^{T}$ and $g_{i}^{(k-1)}=a_{i j} x_{j}^{(k-1)}$. With this method, the relaxation parameter for each pixel can be adjusted according to its estimation obtained from the last iteration. The artifacts noise can also be reduced because the relaxation parameter adaptively decreases with the increase of the iterative number. Thus, the adaptive adjustment of the relaxation parameters can improve computation efficiency and imaging quality of the ART algorithm.

\section{Numerical experiments}

\subsection{Experiment 1 (using simulated data)}

In this paper, the primary purpose is to further improve the computation efficiency and the imaging quality of the ART. Therefore, the IART is evaluated by a comparison of the computation efficiency and the imaging quality between the IART and the ART. Before the IART is applied to perform the inversion of IED from real TEC data, it is also necessary to validate the method. For these purposes, a test experiment is devised here. In this scenario, a set of IED distributions corresponding to 10:40-11:00LT, 19 August 2003 (02:40-03:00UT, 19 August 2003) is provided by the IRI2001 model, and precise known positions of GPS satellites and ground receivers selected from Crustal Movement Observation Network of China (CMONOC) during the observation period of interest are used for establishing coefficient matrix A.

Using the above IED distributions, a set of simulated TEC data are produced for 13 receivers located in China to test the IART method, and then a small amount of random noise is added to the simulated TEC data. The maximum noise is $5 \%$ of the average value of the simulated TEC data. In this simulation, there are 4256 ray paths. It should be noted that the number of ray paths varies with that of visible satellites in the sky at the same time. In addition, a set of initial IEDs is usually required for performing the two tomographic algorithms. In this work, the initial IEDs are set to 0.8 times of the above IED distributions and move $50 \mathrm{~km}$ upward in height. 

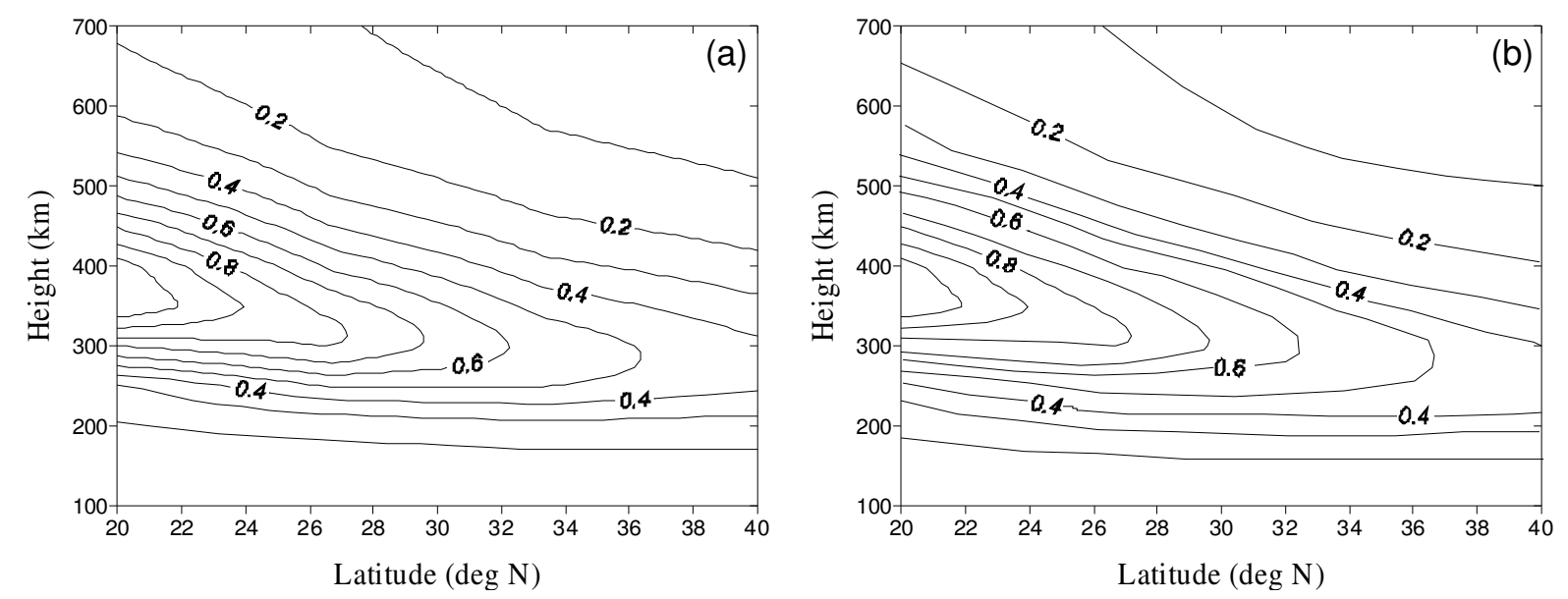

Figure 1. Contour plots of the modeled and the reconstructed ionspheric electron density distribution at longitude $110^{\circ} \mathrm{E}$, and IED is expressed in the unit of $10^{12} \mathrm{e} / \mathrm{m}^{3}$. (a) Modeled IED distribution with the IRI2001 model; (b) Reconstructed IED distribution with the new algorithm (IART).

Figure 1(a) shows the IED distribution on 19 August 2003 at 11:00h local time along a fixed longitude meridian of $110^{\circ} \mathrm{E}$, which is obtained from the IRI2001 (Bilitza, 2003) model. The reconstructed IED image with the IART is shown in Figure 1(b). It can be seen that the equatorial ionization anomaly is reconstructed particularly well. The average density error is $9.5 \times 10^{9} \mathrm{e} / \mathrm{m}^{3}$, which is very small compared with the typical peak electron density of $1.1 \times 10^{12} \mathrm{e} / \mathrm{m}^{3}$. It proves that the IART is feasible to perform ionospheric tomography with a sufficiently high accuracy by using the simulated TEC data. A comparison of convergence performance between the IART and ART technique is shown in Figure 2.
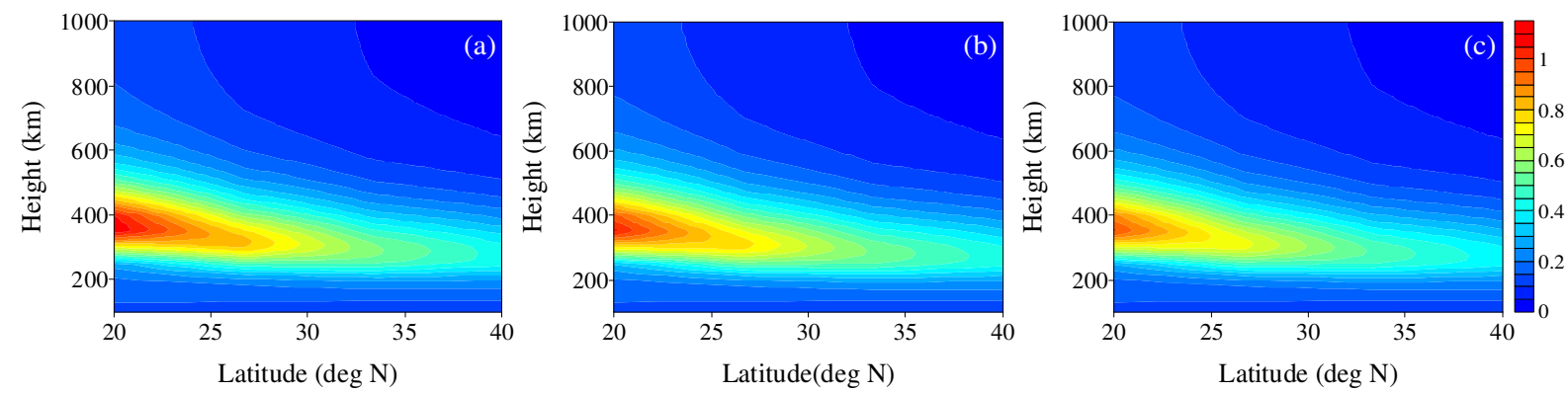

Figure 2. A IED image obtained from numerical ionospheric model and two reconstruction process of five-iteration by the two algorithms. The unit of IED is $10^{12} \mathrm{e} / \mathrm{m}^{3}$. (a) Original IED image with the IRI 2001 model; (b) Reconstructed image with the IART algorithm; (c) Reconstructed image with the ART algorithm.

For simplicity of description, in the following section, one-iteration means that all available ray paths are used to update the reconstruction once. To compare the convergence performance of the IART and the ART, before the convergence of the iteration, the two-dimensional images along a fixed longitude meridian of $110^{\circ} \mathrm{E}$ obtained from the IRI2001 model is compared with those reconstructed from five-iteration results with the IART and the ART, which are shown in Figures 2(b)-2(c), respectively. The two reconstructed images illustrate that the IART has the quicker convergence and the better reconstruction quality. 
Table 1 Error statistics of the reconstructed IED based on two algorithms by using simulated data

\begin{tabular}{|c|c|c|c|c|c|c|}
\hline Geographic locations & \multicolumn{2}{|c|}{$\left(104^{\circ} E, 26^{\circ} N\right)$} & \multicolumn{2}{|c|}{$\left(110^{\circ} \mathrm{E}, 30^{\circ} \mathrm{N}\right)$} & \multicolumn{2}{|c|}{$\left(116^{\circ} \mathrm{E}, 36^{\circ} \mathrm{N}\right)$} \\
\hline Reconstruction methods & ART & IART & ART & IART & ART & IART \\
\hline Maximum of IED errors $\left(10^{10} \mathrm{e} / \mathrm{m}^{3}\right)$ & 7.08 & 3.51 & 8.52 & 3.74 & 2.60 & 1.19 \\
\hline Minimum of IED errors $\left(10^{10} \mathrm{e} / \mathrm{m}^{3}\right)$ & -9.93 & -6.36 & -7.51 & -2.42 & -6.76 & -1.07 \\
\hline Average of IED errors $\left(10^{10} \mathrm{e} / \mathrm{m}^{3}\right)$ & 0.64 & 0.34 & 0.43 & 0.13 & -0.28 & -0.12 \\
\hline Variance $\left(10^{20}\left(\mathrm{e} / \mathrm{m}^{3}\right)^{2}\right)$ & 13.7 & 4.58 & 12.3 & 4.24 & 8.85 & 2.01 \\
\hline
\end{tabular}
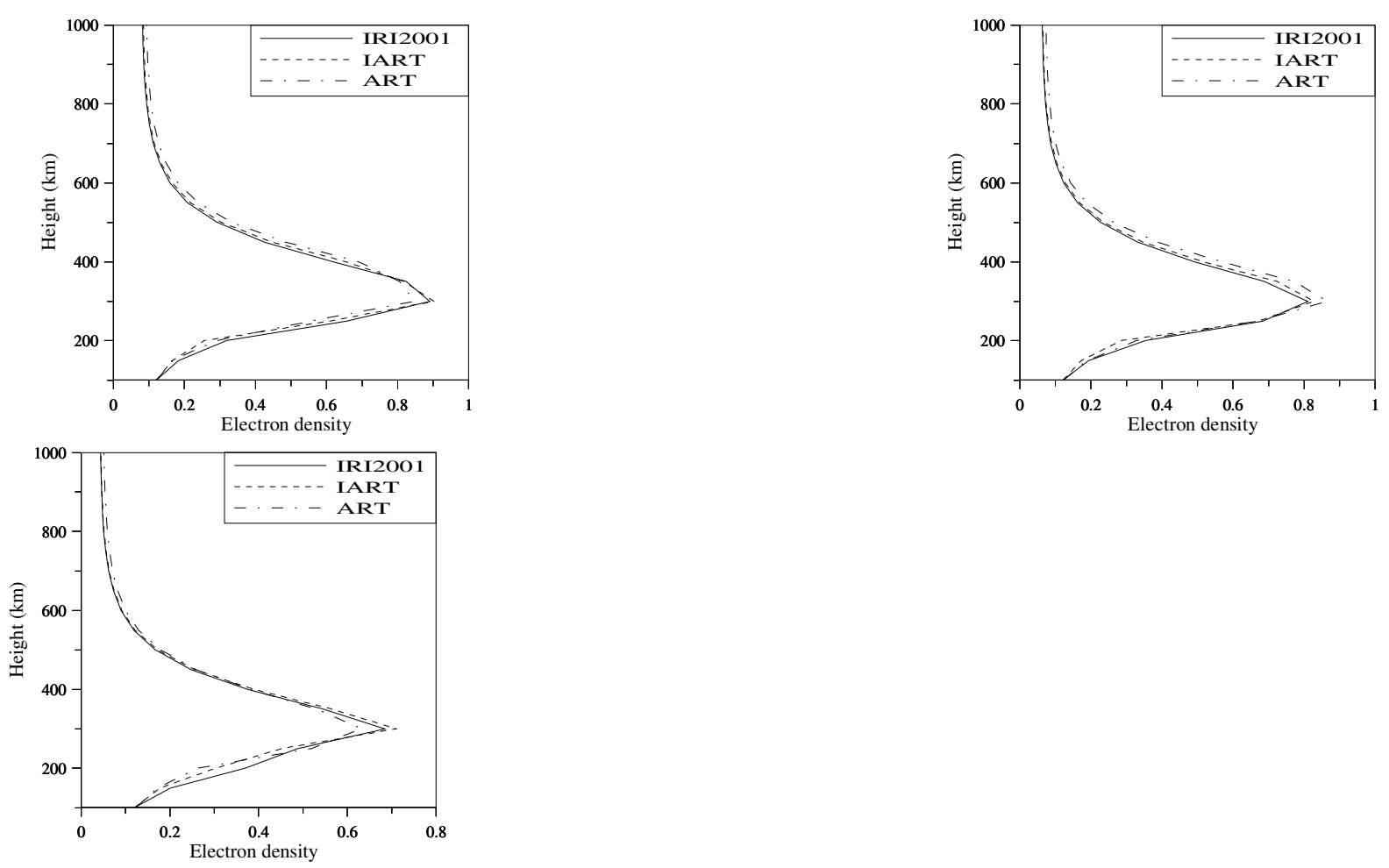

Figure 3. A Comparison among the modeled IED profiles with the IRI2001 model and the reconstructed IED profiles by the IART and the ART at the following geographic locations (a) $\left(104^{\circ} \mathrm{E}, 26^{\circ} \mathrm{N}\right)$, (b) $\left(110^{\circ} \mathrm{E}, 30^{\circ} \mathrm{N}\right)$, and (c) $\left(116^{\circ} \mathrm{E}, 36^{\circ} \mathrm{N}\right)$. The IED is in the unit of $10^{12} e / \mathrm{m}^{3}$.

Figures 3(a-c) compare the simulated vertical IED distributions at the three geographic positions $\left(104^{\circ} \mathrm{E}, 26^{\circ} \mathrm{N}\right),\left(110^{\circ} \mathrm{E}, 30^{\circ} \mathrm{N}\right)$ and $\left(116^{\circ} \mathrm{E}, 36^{\circ} \mathrm{N}\right)$ with the corresponding reconstruction results by using the IART and the ART. A comparison is made through the differences between the results obtained from the two methods and that obtained from the IRI2001 model. Table 1 lists the errors statistics of the inverted IED at the above geographic positions by using the simulated TEC data. From Table 1 and Figure 4, one can see that the IED profiles obtained from the IART agree better with the IRI2001 model than those obtained from the ART as a whole. This indicates that the IART is superior to the ART in performing ionospheric tomography. Similar results are also obtained with the modeled data for different solar activities, seasons and local time.

\subsection{Experiment 2 (using real TEC data)}

With TEC data derived from dual-frequency GPS observations, the IART was applied for the inversion 
of IED. In this work, dual-frequency GPS data was obtained from 13 permanent GPS stations in China, and the sample interval is 30 seconds. To study hourly variation of IED, GPS observations within 20 minutes in every hour are analyzed, and the inversion is carried out in the earth-fixed reference system. Since the vertical resolution of ionospheric tomography is usually very limited due to the insufficiency of horizontal ray paths from GPS satellites to the ground receivers, CHAMP related occultation observations are incorporated into this inversion as much as possible in order to improve the vertical resolution.

Corresponding to ionospheric heights, areas of data assimilation are bounded at the bottom level of $100 \mathrm{~km}$ and up level of $1000 \mathrm{~km}$ in height. Thus, the plasmasphere is not included in the reconstruction process. However, GPS satellites are orbiting the Earth at an altitude of $20,200 \mathrm{~km}$ approximately and the transmitted electromagnetic waves traverse through the plasmasphere before they are received on the ground or onboard LEO satellites at altitude of $400 \mathrm{~km}$ for CHAMP. Plasmasphere electron densities are of the order $10^{8} \mathrm{e} / \mathrm{m}^{3}$, which is $3 \sim 4$ orders smaller than the electron densities in ionosphere. Nevertheless, the plasmasphere may contribute to TEC measurements along GPS rays significantly (Lunt et al. 1999). To estimate the contribution of plasmasphere to the observed TEC value, a simple model proposed by Angerami and Thomas (1964) is used in this work. According to this model, the electron density is assumed decrease exponentially with height, so the electron density distribution $n(h)$ from $1000 \mathrm{~km}$ to $20,200 \mathrm{~km}$ in height is expressed as:

$$
n(h)=n\left(h_{0}\right) \exp \left(-\frac{h-h_{0}}{H_{s}}\right)
$$

where $n\left(h_{0}\right)$ is the electron density at the top of the ionospheric region and $H_{s}$ is the scale height of the density decay in the plasmasphere. Although the electron density of the plasmasphere is distributed along geomagnetic field lines and the scale height $H_{s}$ varies with the season of solar activities, local time and other factors. For simplicity, the value is fixed at the height of 2000km (Reinisch et al. 2001; Leitinger et al. 2002). This is reasonable because the aim of this work is to reconstruct the electron density profile of the ionospheric region, where the contribution of the plasmaspheric region to the measured $S T E C_{m}$ along the corresponding ray path is considered to be very small. Under this assumption, the plasmaspheric contribution to the $S T E C_{m}$ along the ray path of each GPS satellite-receiver pair is given as

$$
S T E C_{p l}=\frac{1}{\cos \theta}=\int_{h_{0}}^{h_{s a t}} n\left(h_{0}\right) \exp \left(-\frac{h-h_{0}}{H_{s}}\right) d h \approx \frac{1}{\cos \theta} n\left(h_{0}\right) H_{s}
$$

where $h_{\text {sat }}$ is the altitude of each GPS satellite and $\theta$ is the inclination of the ray path with respect to the vertical direction. To obtain accurate IED distribution, the STEC used in ionospheric tomography system should be the difference between $S T E C_{m}$ and $S T E C_{p l}$. 

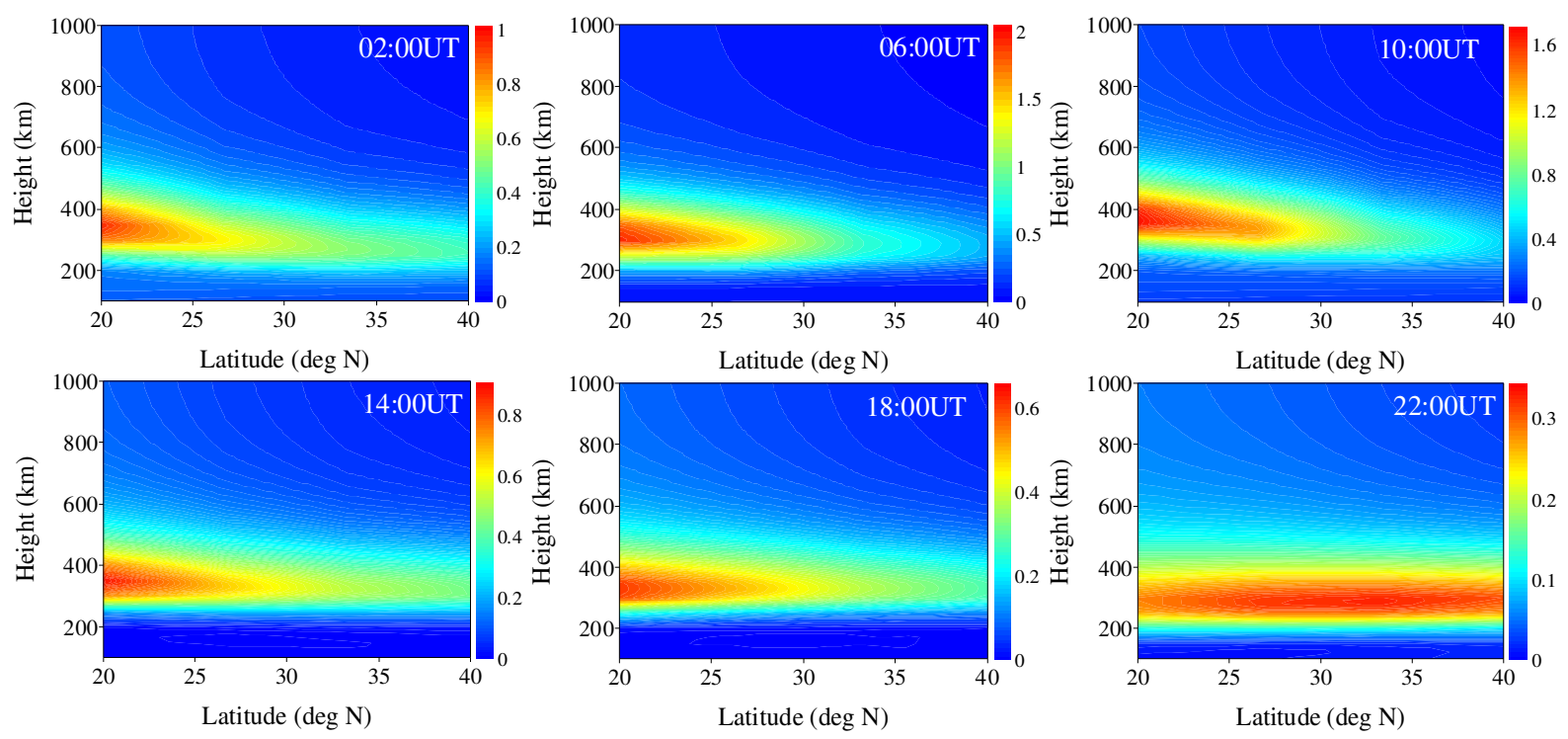

Figure 4. Tomographic reconstruction images using ground-based and space-based occultation GPS data on 19 August 2003. Each image represents the corresponding IED reconstructed in different universal time epochs. The IED is expressed in the unit of $10^{12} e / \mathrm{m}^{3}$

Figure 4 illustrates the hourly variation of the IED along a fixed longitude meridian of $110^{\circ} \mathrm{E}$. From this figure, it can be seen that there are lager differences between the characteristics of ionospheric electron densities in mid-latitude and low-latitude areas, and the values of ionospheric electron densities in the north are smaller than those in the south as a whole. This indicates that there is a strong correlation between the property of IED and latitude. Comparing all subfigures in Figure 4, it can also be seen that the peak height of the IED gradually increases during the time period from 2UT to $10 \mathrm{UT}$, and then it began to fall during the next time period, the peak height of the IED falls to $270 \mathrm{~km}$ at $22 \mathrm{UT}$. This reflects the characteristics of vertical variations of the IED for the day. Figure 5 shows contour plots of the longitude-height cross section at $30^{\circ} \mathrm{N}$ in latitude obtained from 14UT.

To validate the reliability of the vertical resolution of the IART, IED profiles reconstructed with the IART and the ART are compared with those obtained from ionosonde data. In this research, ionosonde data from Wuhan station is used, and a comparison is shown in Figure 6. Table 2 shows the error statistics of the reconstructed IED by using the real TEC data. Table 2 and Figure 6 show that the recovered reconstructed IED profiles from the IART agree better with those obtained from ionosonde at Wuhan station than those recovered from the ART as a whole. Hence, it is suggested that a reliably vertical density distribution may be reconstructed by using the IART. In addition, the comparison between the IART and the ART also shows that the IART is superior to the ART algorithm as a whole.

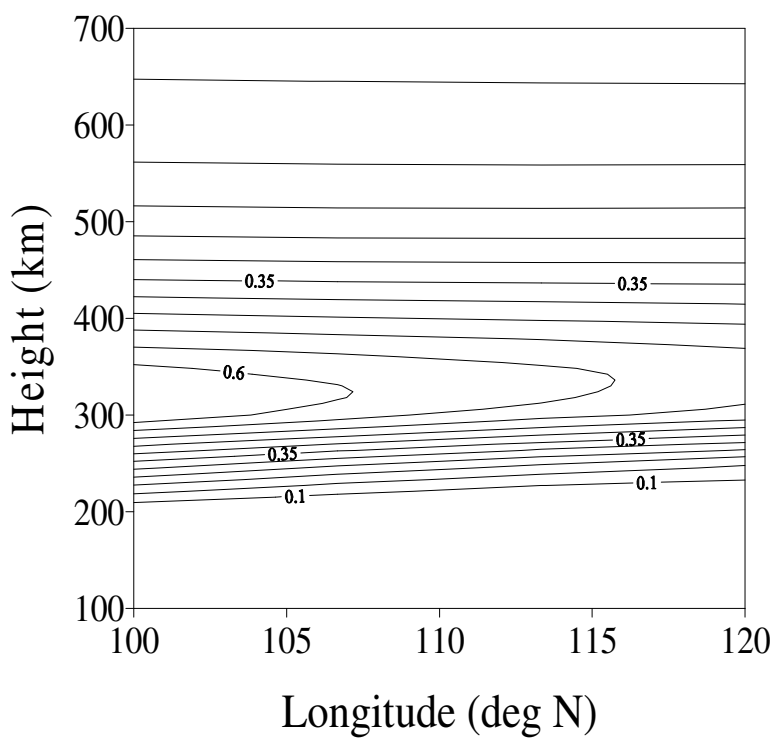


Figure 5. Contour plot of IED (in units of $10^{12} \mathrm{e} / \mathrm{m}^{3}$ ) distribution in a longitude-altitude plane.
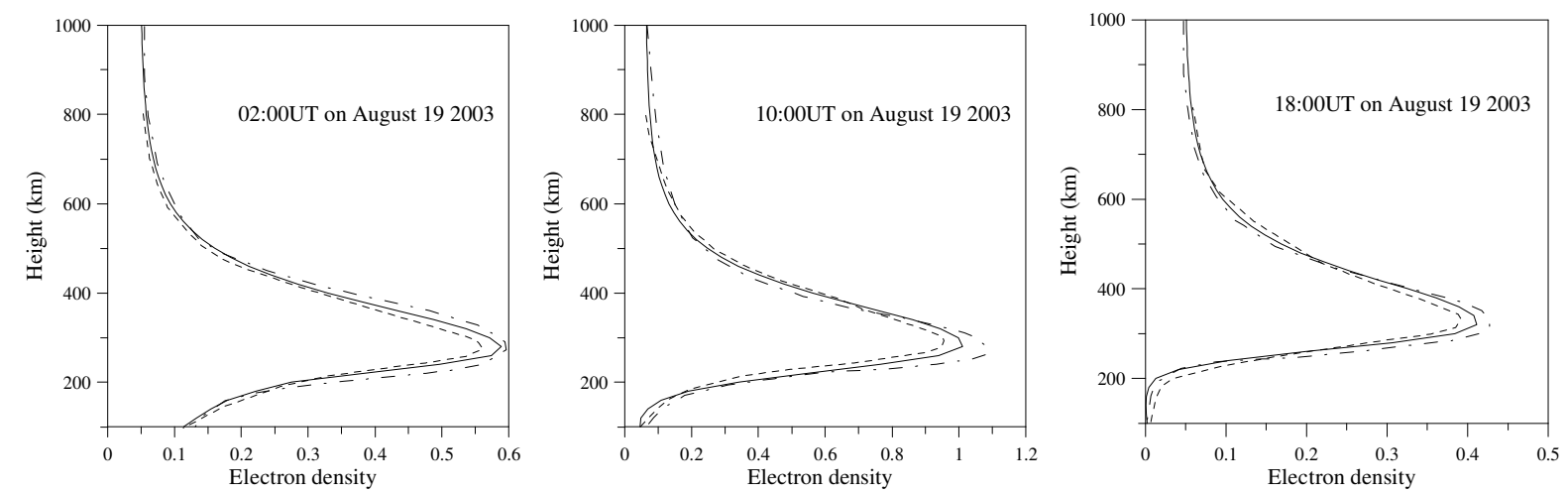

Figure 6 Comparisons of IED profiles obtained from the IART (solid line), ionosonde data (dash line) and the ART (dash dot line). The IED is express in units of $10^{12} e / m^{3}$.

Table 2 Error statistics of the reconstructed IED based on ART and LART algorithms using real GPS data

\begin{tabular}{|l|c|c|c|c|c|c|}
\hline Time (UT) & \multicolumn{2}{|c|}{$02: 00$} & \multicolumn{2}{c|}{$10: 00$} & \multicolumn{2}{c|}{$18: 00$} \\
\hline Reconstruction methods & ART & IART & ART & IART & ART & IART \\
\hline Maximum of IED errors $\left(10^{10} \mathrm{e} / \mathrm{m}^{3}\right)$ & 5.22 & 2.95 & 13.0 & 5.98 & 4.16 & 2.28 \\
\hline Minimum of IED errors $\left(10^{10} \mathrm{e} / \mathrm{m}^{3}\right)$ & -3.68 & -2.90 & -9.13 & -1.98 & -2.46 & -1.87 \\
\hline Average of IED errors $\left(10^{10} \mathrm{e} / \mathrm{m}^{3}\right)$ & 0.46 & 0.21 & 0.97 & 0.35 & 0.36 & 0.10 \\
\hline Variance $\left(10^{20}\left(\mathrm{e}^{\mathrm{m}} \mathrm{m}^{3}\right)^{2}\right)$ & 6.24 & 1.83 & 7.23 & 2.87 & 4.24 & 1.13 \\
\hline
\end{tabular}

\section{Error analysis}

Although the ionospheric image can be satisfactorily reconstructed with the IART algorithm, there exists difference between the true image and the reconstructed image. Hence, it is necessary to find the reasons why the difference occurs. In general, any tomographically reconstructed image is composed of three components: true image, error and artifacts. The true image is the desired image, and it can only be produced when the complete projection data is obtained. There is a difference due to the fact that there exists errors and artifacts. There are various error sources such as experimental measurement error, the errors caused by various approximations and assumptions made on the projection operator, discretized error of ionospheric region, and the error induced from the idealized geometry or conditions, etc.

As mentioned above, GPS-based ionospheric tomography with the IART applies the STEC to invert IED. The reconstruction accuracy of the IART mainly depends on that of STEC. Since the STEC is obtained from the difference between the carrier phases and the difference between the pseudoranges of the two GPS signals in this work, the clock errors of GPS satellites and receivers cannot be taken into account. Then the difference between the true image and the reconstructed image with the IART is partly attributed to the GPS observation errors such as data noise, cycle slips, multipath effects and instrumental bias, etc. The errors exist no matter how the GPS data is processed.

\section{Conclusion}

A new reconstruction technique of IED, termed IART, is proposed in this work. The IART develops a data-driven adjustment for relaxation parameters. It overcomes the disadvantags of the ART to some extent. The performance of the new method has been demonstrated using simulated data generated by the IRI2001 model. Comparisons are made between the IART and the ART. It has been found that the IART can further improve the reconstructed image of the IED and has a better convergence performance. The inverted 
results also demonstrate that the IART is superior to the ART in constructing the ionospheric tomography with high-resolution and high-efficiency. The reconstruction results with the IART agree well with those from ionosonde observation data obtained at Wuhan station. The reliability of the IART in representing the ionospheric tomography is confirmed. In this work, a set of three-dimensional images of IED are successfully reconstructed. In the future, this method is expected to extend to a four-dimensional tomography by considering temporal variations of the ionosphere.

Acknowledgements The authors would like to thank data center for the Crustal Movement Observation Network of China and Shanghai Astronomical observatory of Chinese Academy of Sciences for providing the ground-based GPS tracking station data. Professor Libo Liu from the Institute of Geology and Geophysics of Chinese Academy of Sciences is also specially thanked for providing ionosonde data. ISDC GFZ provides the CHAMP occultation measurements. This research is supported by the National Science Fund for Distinguished Yong Scholars of China (Grant No. 40625013) and the National Natural Science Foundation of China (Grant No. 40674012 and No. 40474009).

\section{References}

Andersen AH (1989) Algebraic reconstruction in CT from limited views. Med. Imaging 8:50-55

Andreeva ES, Galinov AV, Kunitsyn VE, Melnichenko YA, Tereshchenko E, Filimonov MA, Chernyakov SM (1990) Radio tomography reconstruction of ionostation dip in the plasma near the earth. J Exp. Theor. Phys. Lett 52:145-148

Angerami JJ, Thomas JO (1964) Studies of planetary atmospheres: The distribution of electrons and ions in the Earth's exosphere. J. Geophys. Res. 69:4537-4560.

Austen JR, Franke SJ, Liu CH (1986) Applications of computerized tomography techniques to ionospheric research. In: Radio beacon contribution to the study of ionization and dynamics of the ionosphere and corrections to geodesy. Ed. A. Tauriainin, Oulu, Finland, part 1: pp. 25-32 Bilitza D (2003) International reference ionosphere. http://nssdc.gsfc.nasa.gov/space/model/ionos/iri.html Blewitt G (1990) An automatic editing algorithm for GPS data. Geophys. Res. Lett. 17:199-202 Breed AM, Goodwin GL, Vandenberg A-M, Essex EA Lynn KJW, Silby JH (1997) Ionospheric total electron content and slab thickness determination in Australia. Radio Sci. 32(4):1635-1643.

Bust GS, Coco D, Makela JJ (2000) Combined ionospheric campaign 1: Ionospheric tomography and GPS total electron content (TEC) depletions. Geophys Res Lett 27(18): 2849-2852

Fremouw EJ, Secan JA, Howe BM (1992) Application of stochastic inverse theory to ionospheric tomography. Radio Sci 27:721-732

Hajj GA, Ibanez-Meier R, Kursinski ER, Roman LJ (1994) Imaging the ionosphere with the Global Positioning System. Int J Imag Syst Technol 5(2): $174-184$

Hansen AJ, Walter T, Enge P (1997) Ionospheric correction using tomography. In: Proceedings of the institute of navigation. GPS-97, pp.249-257 Hernandez-pajares M, Juan JM, Sanz J (1998) Global observation of ionospheric electronic response to solar events using ground and LEO GPS data. J Geophys Res 103(A9): 20789-20796

Horvath I Essex EA (2003b) The southern-hemisphere mid-latitude day-time and nighttime trough at low-sunpot numbers. J. Atmos. Terr. Phys. 65:917-940.

Howe BM, Runciman K, Secan JA (1998) Tomography of the ionosphere: Four-dimensional simulations. Radio Sci 33(1): 109-128 Jakowski N, Sardon E, Engler E, Jungstand A, Klahn D (1996) Relationship between GPS-signal propagation errors and EISCAT observations. Ann Geophys 14:1429-1436

Klobuchar JA (1996) Ionospheric effects on GPS. In: Parkinson BW and Spilker JJ, Global Positioning System: Theory and Application Vol.1. 
American Institute of Aeronautics and Astronautics INC.

Kunitake M, Ohtaka K, Maruyama T, Tokumaru M, Morioka A, Watanabe S (1995) Tomographic imaging of the ionosphere over Japan by the modified truncated SVD method. Ann Geophys 13:1303-1310

Kunitsyn EV, Tereshchenko ED (1992) Radio tomography of the ionosphere. IEEE Antennas Propag. Mag 34:22-

Leiginger R, Ladreiter HP, Kirchengast G (1997) Ionospheric tomography with data from satellite reception of Global navigation satellite system signals and ground reception of navy navigation satellite system signals. Radio Sci 11(1): 1657-1669

Leitinger R, Radicella S,. Hochegger G, Nava B (2002) Diffusive equilibrium models for the height region above the F2 peak. Adv. Space Res. 29(6):809-814.

Lunt N, Kersley L, Bishop, G. J, Mazzella Jr, A. J., Bailey, G. J. (1999). The protonospheric contribution to GPS total electron content: Two-station measurements. Radio Sci., 34(5):1281-1285.

Ma XF, Maruyama T (2005) Three-dimensional Ionospheric tomography using observation data of GPS ground receiver and ionosonde by neural network. J Geophys Res Vol.110. A05308. DOI 10.1029/2004JA010797

$\mathrm{Na} \mathrm{H}$, Lee H (1990) Resolution analysis of tomographic reconstruction of electron density profiles in the ionosphere. Int J Imag Syst Technol 2: $209-218$

Oskoui P, Stark H (1989) A comparative study of three reconstruction methods for a limited-view computer tomography problems. IEEE Trans. Med. Imaging 8:43-49

Pryse SE, Kersley L (1992) a preliminary experimental test of ionospheric tomography. J. Atmos. Terr. Phys 54:1007-1023

Raymund TD, Austen JR, Franke SJ, Liu CH, Klobuchar JA, Stalker J (1990) Application of computerized tomography to the investigation of ionospheric structure. Radio Sci 25: 771-789

Reinisch BW (2001) First results from the radio plasma on IMAGE. Geophys. Res. LEtt. 28:1167-1170.

Rius A, Ruffini G, Cucurull L (1998) Improving the vertical resolution of ionospheric tomography with GPS occultation. Geophys Res Lett 24(18): $2291-2294$

Ruffini G, Flores A, Rius A (1998) GPS tomography of the ionospheric electron content with a correlation functional. IEEE T Geosci Remote 36(1): $143-153$

Sardon E, Rius A, Zarraoa N (1989) Estimation of the receiver differential biases and the ionospheric total electron content from Global Positioning System observations. Radio Sci. 29:577-586.

Stolle C, Schluter S, Jacobi C, Jakowski N (2003) 3-dimensional IED reconstruction based on GPS measurements. Adv Space Res 31(8). DOI $10.1016 / \mathrm{S} 0273-1173(03) 00168-6$

Wu XB, Xu JS, Ma SY (2000) An improved reconstruction algorithm for computerized ionospheric tomography. Chinese J Geophys-CH 43(1): 19-28 Yin P, Mitchell CN, Spencer PSJ, Foster JC (2004) Ionospheric electron concentration imaging using GPS over the USA. Geophys Res Lett 31 , L12806. DOI 10.1029/2004GL019899

Yizengaw E, Dyson PL, Essex EA, Moldwin MB (2005) Ionosphere dynamics over the southern Hemisphere during the 31 March 2001 severe magnetic storm using multi-instrument measurement data. Ann Geophys 23:707-721

Yizengaw E, Essex EA, Brisa R (2004) The souther hemisphere and equatorial region ionization response for a 22 septemer 1999 severe magnetic storm. Ann Geophys 22:2765-2773

Figure 1. Contour plots of the modeled and the reconstructed ionspheric electron density distributions at longitude $110^{\circ} \mathrm{E}$. IED is expressed in units of $10^{12} \mathrm{e} / \mathrm{m}^{3}$. (a) Modeled IED distribution with the IRI2001 model, (b) Reconstructed IED distribution using the new algorithm proposed (IART).

Figure 2. Numberical ionospheric model and images reconstructed through five-iteration by the two 
algorithms proposed. (a) Original IED image from the IRI 2001 model; (b) Reconstructed image using the IART algorithm; (c) Reconstructed image using the ART algorithm.

Figure 3. A Comparison among the modeled IED profiles with the IRI2001 and the reconstructed IED profiles by the IART and the ART methods at the geographic positions (a) $\left(104^{\circ} \mathrm{E}, 26^{\circ} \mathrm{N}\right)$, (b) $\left(110^{\circ} \mathrm{E}, 30^{\circ} \mathrm{N}\right)$, (c) $\left(116^{\circ} \mathrm{E}, 36^{\circ} \mathrm{N}\right)$. The unit of IED is $10^{12} \mathrm{e} / \mathrm{m}^{3}$.

Figure 4. Tomographic reconstruction images using both ground-based and space-based occultation GPS data on 19 August 2003. Each subfigure represents the corresponding IED reconstructed in different universal time epochs.

Figure 5. Contour plots of IED (in units of $10^{12} \mathrm{e} / \mathrm{m}^{3}$ ) distribution in a longitude-altitude plane.

Figure 6 A comparison of IED profiles obtained from the IART (solid line), ionosonde data (dash line) and the ART (dash dot line). The IED is express in units of $10^{12} \mathrm{e} / \mathrm{m}^{3}$. 